\title{
Managing the Digital Knowledge Work with the Social Media Business Value Compass
}

\author{
Kathrin Kirchner \\ Technical University of Denmark \\ kakir@dtu.dk
}

\author{
Liana Razmerita \\ Copenhagen Business School \\ 1ra.msc@cbs.dk
}

\begin{abstract}
Companies are increasingly adopting social platforms for supporting their digital workforce, which is more and more virtual or dispersed in crossfunctional teams across different locations. Supporting the social processes (communication and collaboration between employees) become an essential task associated with the future of the work. Organizations need reliable measures to understand social platform usage, their effect on knowledge work and ultimately to increase the derived business value. Understanding how to assess the perceived business value would help managers to optimize the usage and increase the impact of digital social platforms at work. This paper proposes the social media business value compass as a tool that enables managers to assess the perceived business value derived from the usage of enterprise social platforms. It classifies the business value along four dimensions: efficiency, innovation, retention and transparency. Using the social media business value compass, managers can redefine and orient their digital strategy.
\end{abstract}

\section{Introduction}

As part of their digital transformation, businesses, especially in global multinational organizations, are increasingly adopting enterprise social platforms in order to streamline the diversity of knowledge work processes (in particular communication and collaboration) or engage and coordinate with partners across geographical and cultural boundaries. According to McKinsey [2], some of the digital initiatives generate attractive returns, while others don't return their cost of capital. Managers need strategies to deal with this distributed, diverse and mobile digital workforce, including strategies for managing a growing number of aging employees and skill drain.

Enterprise social platforms are social mediaenhanced platforms that support communication, colla- boration and networking such as Yammer, Chatter, Slack, IBM connections, or collaborative work and project management such as Podio, Asana, Jira, Microsoft Teams or Trello. These digital platforms support both formal and informal communication, collaboration in virtual teams and distributed work arrangements and are therefore heralded to become the future of work. Furthermore, they support more informal, flexible working environments and include features similar to mainstream social networking applications (e.g. Facebook@Work), and we shall therefore, in this article, name them enterprise social platforms. In the literature, they are also referred to as Social Media at Work, Enterprise Social Networks or Enterprise Social Media as they may integrate a variety of social media tools such as social networks, blogs, wikis, and content communities. Although platforms of this type may facilitate the management of social networks and access to digitized content, they may also impact both employees' behavior and employees' performance. However, managers "should be wary of assuming that performance effect will be positive and they should beware of possible unintended outcomes"'[7].

Executives have recognized that potential enterprise social platforms have to strengthen company communication, collaboration and knowledge sharing with both internal and external stakeholders and improve strategic decisions. According to a McKinsey report, the deployment of enterprise social platforms has taken place in three phases [5]. In the first phase, in the mid of the 2000s, companies started to use social platforms, especially in marketing (e.g., by using Facebook, Twitter or YouTube) to get in touch with customers. In the second phase (from around 2010), companies started using internal social platforms (e.g. Yammer and Chatter) to collaborate and build connections between employees. In the third phase (from 2014), social platforms have started to be used for building strategies and opening up for a company-wide collaboration and communication.

Most of the consultancy reports discuss the business value of enterprise social platforms taking into account the top management perspective, but we shall 
here illustrate the perceived business value of social platforms from the middle management perspective rather than the top management perspective. Organizations need valid, reliable measures to understand social platform usage, their effect on knowledge work and ultimately to increase the derived business value [10]. According to our case studies investigated over several years, many companies are not measuring the benefits or assessing the business value of their enterprise social platforms. Understanding how to assess the perceived business value of enterprise social platforms would help managers to optimize the usage and increase the impact of social platforms at work.

The business value compass proposed in this paper was created based on a series of interviews that we conducted with middle managers and CEOs of four knowledge-intensive organizations ranging from small multinational IT start-ups to big multinational IT consultancies. All these organizations have adopted enterprise social platforms to support knowledge work tasks for their digital workforce. The companies use a variety of digital tools including Slack for internal communication across departmental boundaries, Asana as a project management tool, or Podio for collaboration among the teams.

The interview guideline included questions related to the type of tools in use, their usage in different departments and teams, perceived benefits and challenges. Furthermore, we address issues related to key factors influencing the adoption of social media including the strategy, the business value assessment, its impact and associated transformations in the organization. The collected data was coded in NVivo using open, axial and selective coding technique. This analysis was combined with previous research findings $[14,16]$, in order to derive the four dimensions of business value: Efficiency, Innovation, Retention and Transparency. Based on the analysis of 12 interviews, we extracted sub-concepts and categories associated with the business value which were further mapped into the four dimensions. These dimensions constitute the basis for the Social Media Business Value (SMBV) compass (as represented in Figure 1). The SMBV compass was evaluated with two IT consultancies and a non-profit organization that were not involved in the initial data collection. Based on this evaluation some sub-concepts were renamed. Our SMBV compass helps managers to orient their digital strategy in order to enhance their business value, especially in the areas where they do not derive enough value or the specific area they would like to focus on. However, in order to reap the benefits and associated business value companies have to overcome a number of potential challenges associated with the adoption of social platforms.

\section{Challenges for Social Platforms}

By democratizing communication using enterprise social platforms, all employees can contribute freely and business value can be increased. However, there are also risks and challenges associated with the use of enterprise social platforms.

Below we briefly discuss the main risks that need to be considered based on previous research and our qualitative data analysis. Among the most prominent risks identified in previous studies are: inappropriate behavior and content either deliberate or inadvertent (e.g. off-topic messages, trolls, provocative, alienating or irrelevant messages, out of line with organizational goals), initiating non-work-related conversations or unpleasant conversation, or leak of sensitive knowledge. Managers, but also informal leaders, or co-workers on the platform can intervene to mitigate inappropriate behavior or content [12].

Such a potential risk of leak of sensitive information has been pointed out in our interviews. Because knowledge is accessible to everyone, it could also be misused: "For example interns have access and they are only here for 3-4 months. Tomorrow they can download something and they can misuse it."

Furthermore, the integration of social platforms in the daily routines of employees in the whole company is also connected with challenges at both individual and organizational levels [13]. An important challenge for companies is the engagement of employees on such platforms. A study of factors that impact social media communication in organizations found that the most significant barriers for knowledge sharing at the individual level are: lack of trust in colleagues and fear that knowledge might be misused, lack of time. At the organizational level, an important challenge is associated with a need to change the behavior of employees from hoarding to sharing knowledge. The study found that both intrinsic and extrinsic motivational factors ranging from promotion, monetary rewards, increased social capital or reputation (soft capital), including the recognition of knowledge sharing behaviors, should be considered in order to overcome the social dilemma of sharing knowledge. A set of associated interventions have been proposed in the literature to change the employees' position from a free rider to a cooperative strategy [15].

On social platforms, employees might decide to act like givers or takers. As a giver, they share knowledge or make contributions. If they decide to act like a taker, they like other people to provide knowledge and to support them, but they hide their own expertise [4].

Information overflow and getting lost in the "social" information space (social interaction overload) was another issue that one manager pointed out: "How 
do you feel sure that I get the right information, with the right currency and relevance for me, and can I use it? That's the big trick. It is not the amount of data; we have passed that point. Now it is about making data smart."

People can be online permanently and responsive, and consequently the separation between work and leisure time might become blurred. Some platforms like Slack allow the input of absence times into the system, so that messages are not pushed if the employee does not want to be available: "So there are a lot of challenges in adopting [social platforms] naturally, and even more challenges if you are adopting it saying "I want this percentage to be on this platform actively and what does that by the way mean."

Furthermore, employees may waste their time on social platforms, especially if they use these platforms for non-work related purposes: "[Companies] think that their business productivity is wasted if their employees keep on chatting on these networks."

Several problems can be self-regulated among the employees. One manager reported in the interview that if people are not contributing their knowledge, another colleague could directly ask them why they are not contributing in view of the fact that they are experts on a specific topic. Furthermore, if a person is too often active, tries to answer everyone, and thus wastes time, it can also be regulated by a direct talk among colleagues. Unintended consequences should also be taken into account: e.g. the virtualization of communication may lead to a lack of real social interaction and communication between employees or perhaps more stress instead of more satisfaction.

\section{Social Media Business Value Compass}

As described earlier, SMBV Compass includes four main dimensions: Efficiency, Innovation, Retention and Transparency. Efficiency and Innovation are the main business value factors that every organization wants to increase [9]. Retention and Transparency are two new categories that we derived from our interviews. Both retention of employees and retention of knowledge are important for organizations. Employees that have gained experience over the years work more efficiently and can constitute key assets for organizations. Retention of talent including IT talent is significant "in terms of costs that an organization phases in hiring and training replacements but also in the productivity losses " $[8,11]$.

Retention of knowledge facilitates knowledge reuse, which means that organizations can avoid "to reinvent the wheel". Transparency of communication and knowledge enables employees to access information and be updated, so ideas can flow more quickly, which may also lead to higher innovation performance. In our study, these dimensions were compared with existing theoretical perspectives to build the social media business value compass (as represented in Figure 1). In the following section, we introduce the four dimensions and associated sub-categories. For each sub-category, we include excerpts from the interviews in the subsequent section. Furthermore, in a second phase we tested the SMBV compass in order to get feedback and further insights from its usage.

\subsection{Efficiency}

The most important effect of social platform usage is the increase of efficiency [19]. The platforms may raise the productivity of the digital workforce, e.g., BASF reported an increase of $25 \%$ efficiency of project groups using social platforms. This efficiency gain was possible because new team members had been able to access the whole history of group communication and the decision-making process [7].

Knowledge work processes can be streamlined and this in turn may lead to improved performance and efficiency of employees (e.g. by spending less time writing emails) as extracted from the interview with one of the managers: "It reduces the number of irrelevant emails I have to send when I can post the information in one place."

Knowledge sharing is increased and it is "easier to make a strong relationship between marketing and sales, which is something that a lot of companies are struggling with." Even though virtual work, especially virtual communication, allows for teamwork collaboration and coordination, it does not replace face-to-face interaction: "You have a completely different kind of interaction when you go there and meet the teams face to face".

Such platforms provide the possibility for communicating messages through channels to specific individuals, groups or broadly to all employees.

In addition, communication within the company becomes more efficient and faster in the whole company. A manager pointed this out during an interview: "Via Slack (...) communication is quicker than with email, and you can quickly communicate with a group of people, that makes it easier in a lot of situations." [Without Slack] "I'm sure you will lose at least 2 to 3 times the response time between people."

Another process that may be additionally streamlined is the integration of new employees. By getting access to all the knowledge resources, it is quicker and easier for them to learn how the work is done, to obtain information about ongoing projects or the state of a specific project. 


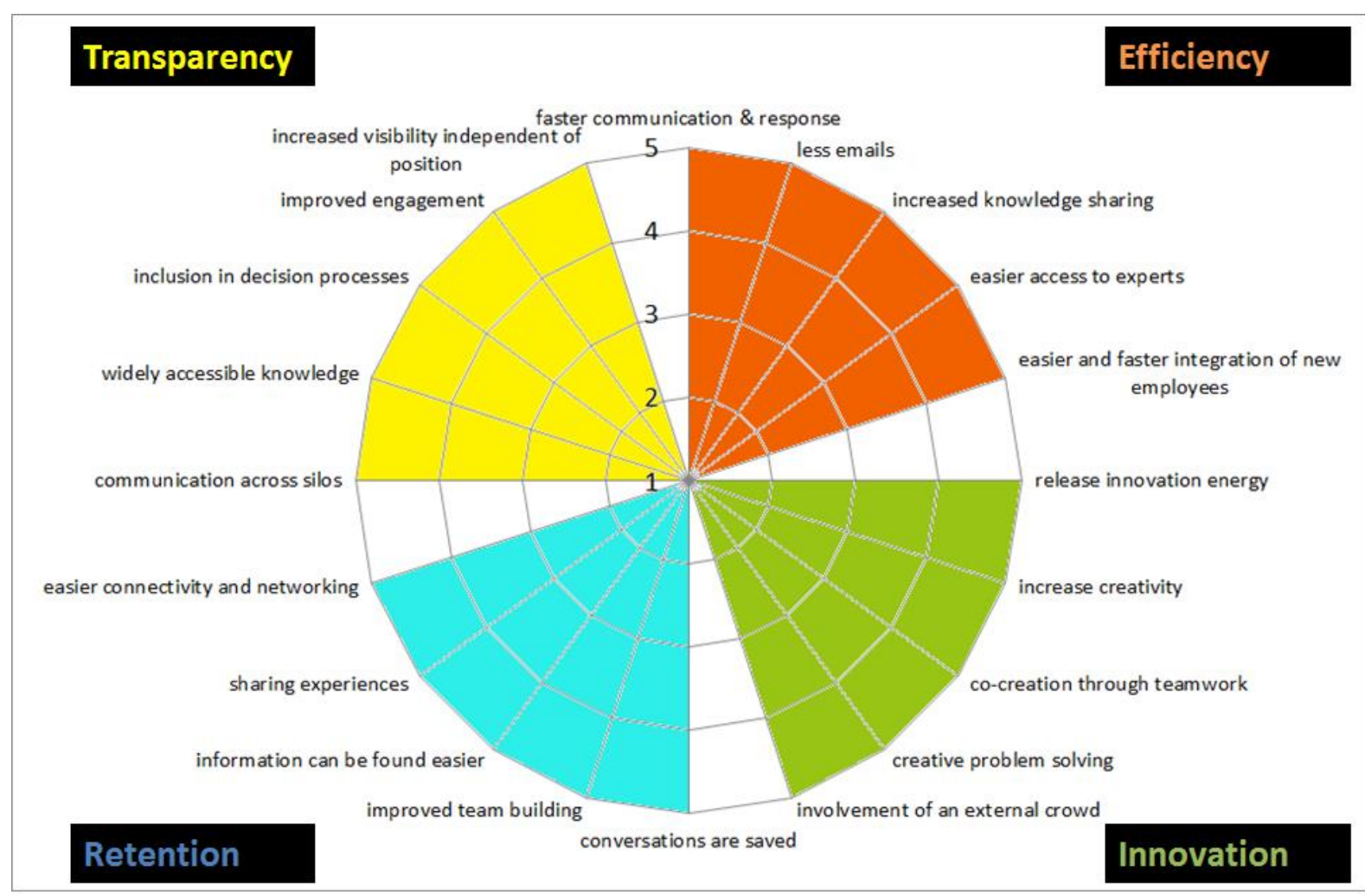

Figure 1: The Social Media Business Value (SMBV) Compass

Further, using social platforms employees can more easily make connections with colleagues and find experts who they can ask for support: "The good thing is if a new employee joins us, [s/] he has access to the whole history of all public channels."

Experts who are knowledgeable in a certain area can be identified more easily because " $a$ lot of the strong ideas come from people that were not identified within that professional area".

Additionally, some respondents mention that traveling costs can be reduced because virtual teamwork can be done in an effective way using various digital tools and enterprise social platforms.

\subsection{Innovation}

The second dimension of the SMBV compass is innovation. Social platforms make it possible to leverage both personal and collective knowledge and thus companies can harness collective intelligence and creativity of the whole digital workforce. Earlier studies have shown that personal and collective knowledge do not compete with each other but they can be reinforced in an effective manner using social media [13]. As these platforms may integrate both crowdsourcing and crowd work features, it is possible to involve both internal and external communities. People can be involved to develop ideas for solving a particular problem using the principles of open innovation. For example, IBM is organizing Innovation Jams across the globe and in this way, they can involve thousands of employees and a diversity of knowledge. "We ask the team across Europe to submit/vote for ideas about what topics would be most interesting / valuable to them." Analytical algorithms make it possible to analyze unstructured data to define the overall sentiment, the influencers, and to predict key concepts and derive recommendations for actions. Solutions can be shared, rated and commented on by others and thus turn a large number of ideas into inventions.

This form of crowdsourcing support leads to innovation processes by allowing everybody to participate and thus lowers the cost of developing new ideas. Furthermore, it opens up the possibility for including creative ideas independent of the job title and level of expertise that would otherwise remain unnoticed: "Social media will give introverted people to have a better chance to come up with ideas. It is not about meeting in the meeting room and talking, it is something you can do at any time. You can post something and share it with the rest of the organiza- 
tion. It enables a lot of ideas that would never come to the table." Thus, "social media releases the personal innovation energy."

\subsection{Retention}

A less emphasized dimension of social media business value is retention. Enterprise social platforms support retention at two different levels: (1) retention of knowledge and digital content and, potentially, (2) retention of the digital workforce. Firstly, informal conversations taking place on digital platforms or answers to questions posed help externalization of tacit knowledge and thus contribute to organizational knowledge (organizational capital). Shared digital content is persistent over time, so it can be searched or retrieved:

"... all the conversation histories are saved and you can go back. I can look for a message that someone sent me back in April last year. And I will find it pretty easily because the search capability is really good."

Enterprise social media enable a capital-creation opportunity. In addition to the contribution to the organizational capital through retention of knowledge, it facilitates connectivity of employees and networking in organizations (social capital), it contributes to informal socialization, learning processes and generates new competencies or skills (human capital).

Secondly, employees feel more connected within their (virtual) teams and their company, and strengthen their relationship with colleagues, and this may lead to the retention of the digital workforce: “...improve staff retention through developing a more social, networked, collaborative organization." Employees feel more part of the company, even when they are working at home or on customer sites. This makes it possible for the company to act more as a global virtual organization. Enterprise Social Media can be associated with an "informal organizational socialization tool" [11].

Additionally, knowledge and experience of employees leaving or retiring are retained and do not get lost. Searching for the right information is also less timeconsuming, as one of our interviewees said: “... a lot of time is wasted on finding information, and that can be optimized by using a social platform like Podio."

Podio allows for context-sensitive searching within tasks, status, conversations or user profiles. Search results can be ranked according to the personal interactions, ratings or popularity of the contributor, interest in a certain workspace or a specific activity.

Sharing of experiences and expertise is easier using social platforms as well as getting feedback and comments on professional discussions: "it gives an opportunity for people who want to share or get feedback, and it gives them a voice in terms of what's going on, and right now."

\subsection{Transparency}

We argue that the value of business social media that is least recognized is transparency and open communication. Enterprise social platforms enable exchange of information across silos, and thus information can flow freely between the departments or across business units distributed across different geographical locations. If a company is open about its activities, from top-level management to all its employees, trust is increasingly built in the company. Trust is important as it is the basis for collaboration and knowledge sharing in organizations. Open communication can lead to improved internal processes and communication across silos: "Now it is super easy to make requests to our support team and talk to IT operations team if you need technical help. It has really changed the way we talk."

Information that would otherwise be hidden in mails can be distributed or shared with the help of social platforms. Public channels enable open communication and exchange of ideas, and it is possible to keep track of activities in the whole company:

"What is important with Slack is that most of the communication is done in public channels in order to use the power of the group, because you might get an answer from someone you didn't have in mind before."

Transparency can also lead to improved employee morale, trust and satisfaction. Especially, if the whole company is willing to share ideas and involve others regardless of the position in the company "it takes away the distinction between what is your title versus what you as a person have in terms of ideas, beliefs and knowledge that you can actually add value to the common good."

Additionally, employees can be involved in conversations, give feedback and thus be involved in the decision-making process: "There is a high interest of employees to know what the CEO is doing and to comment on this, or maybe to post an idea. [Slack] is an easy way to communicate to all employees and helps a lot towards getting more transparent (...) and the CEO started a discussion on Slack on the objectives and key results for 2018, in order to achieve a high level of transparency".

Knowledge and discussions are widely accessible across the subsidiaries: "A developer can have a look at all the announcements from the engineering department, to see how many things happen, what is new, what will happen next". 


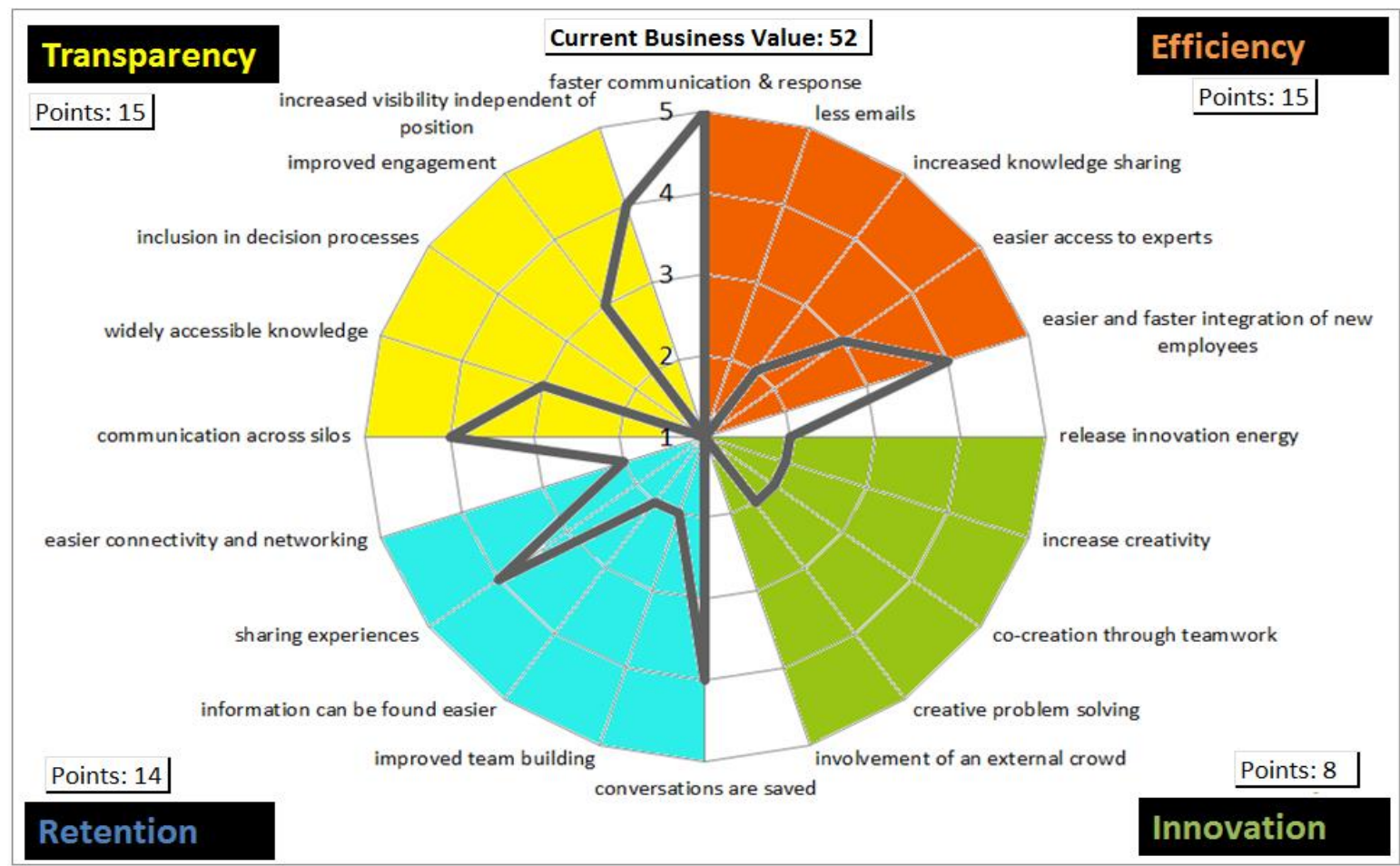

Figure 2: SMBV Compass for a multinational consultancy company

Engagement can be increased by using social media: "I saw that the engagement on the posts on Podio was actually quite high from the people that I expected."

Each employee can set up a communication channel and work with colleagues from all over the company immediately without getting approval from project or department leaders or the installation of a communication tool. From a manager perspective, transparency also involves becoming aware of what tasks employees are working on and how much time they spent on specific tasks, especially when the tasks are associated with billable time for clients. This type of transparency can be achieved by using a "time tracker", which has been implemented in one of the companies we interviewed.

\section{Evaluating the SMBV Compass}

In order to optimize digital knowledge work and the usage of social platforms in an organization, it is important to understand the perceived business value for employees as well as the associated challenges of using the platform. The social media business value compass is shown in Figure 1. The compass comprises the four different dimensions (marked with different colors) introduced in the previous section: Efficiency,
Innovation, Retention and Transparency. As can be seen in Figure 1, each of the four dimensions of the compass comprises five sub-categories. As an example, the efficiency dimension is characterized by the five sub-categories "faster communication and response", "less emails", "increased knowledge sharing", "easier access to experts" and "easier and faster integration of new employees". The compass helps to measure to what extent (on a scale from 1 to 5) social collaborative platforms support the work processes and transformations in a company. In every dimension of the compass, a maximum of 25 points can be obtained. The maximum amount of the estimated value that a company can gain using the business value compass is a score of one hundred points. SMBV compass is a tool that managers and executives can use to estimate and visualize the current business value derived from the usage of social platforms. By using the SMBV compass, companies may become aware of areas that can be further improved.

In order to measure the perceived business value, a questionnaire that covers all four dimensions and their sub-categories (twenty questions in total) was developed. Each question could be answered on a Likert scale $(1 \ldots 5)$. The results from the questionnaire are presented in the SMBV compass. 
In the following, we give two examples of how the SMBV compass was used, based on data elicited from two managers from two different organizations. Figure 2 shows the compass from a company that focuses on consulting and technology implementation. It is a large multinational company with more than 300,000 employees in more than 40 countries. This IT consultancy is hierarchically organized and uses a company-wide social platform for knowledge management and ideation. The aim of the company is to improve the engagement of the employees. Level of engagement is measures using points. Engaged employees can redeem the points they earned on the platform. We have interviewed one of the managers to discuss the perceived business value and the associated results as presented on the SMBV compass.

The business value of social platforms can be quantified with the compass on the level of a single subcategory, on the level of dimension and the level of the whole compass (Figure 2). On the level of a sub-category, the company had already obtained a faster communication and response rate with the help of social media (rated 5 out of 5), but the number of emails did not decrease (rated 1 our 5). Because the platform is used primarily for knowledge management, emails are still the main way to communicate with other employees.

The low values for the innovation were also explained. As sharing innovative ideas is not rewarded in the company, employees do not see the value of contributing yet. In the retention dimension, sharing of experiences and saved conversations are already perceived as a business value, but networking among employees is not supported in a sufficient way among the employees. Regarding transparency, social platforms are not used to include employees on different levels into the decision process. On the level of dimensions, the company achieved the lowest business value in the innovation dimension (rated 8 out of 25). On the overall business value level, the current estimated business value is 52 that is approximately half of the maximum of achievable business value. The SMBV compass was considered very useful for becoming aware of the current usage and perceived business value derived from the social platform. For example, the compass represented in Figure 2 highlights that the performance in the innovation dimension is low. This can be used as a basis for discussions on how to improve this dimension in the future.

The compass can also be used to visualize the business value of social platforms that the company wants to achieve in the future. Figure 3 presents a condensed orthogonal version of the compass showing the current mean values for each of the four dimensions. The business value derived is oriented towards transpa-



Figure 3: Orthogonal version of the compass

rency and retention, while the innovation dimension plays a minor role.

In the future, the company could plan to enhance the business value of their social platform. As can be seen in the example in figure 3, the management of the company decided to achieve especially more business value in the retention and transparency dimension. Here, the improvement is planned to be higher than in the efficiency and innovation dimension.

The business value could also be improved on only one or two dimensions, depending on the strategic aims of the company and the available time. Thus, a company can select which dimension is important for them, or which dimension should be improved first. The condensed compass provides a good visualization of the direction in which the business value should be further improved in the future.

The compass can also be used to compare companies or business units within a company on their usage of social platforms alongside the four different dimensions. Figure 4 gives an example by comparing two companies included in the evaluation of the compass.

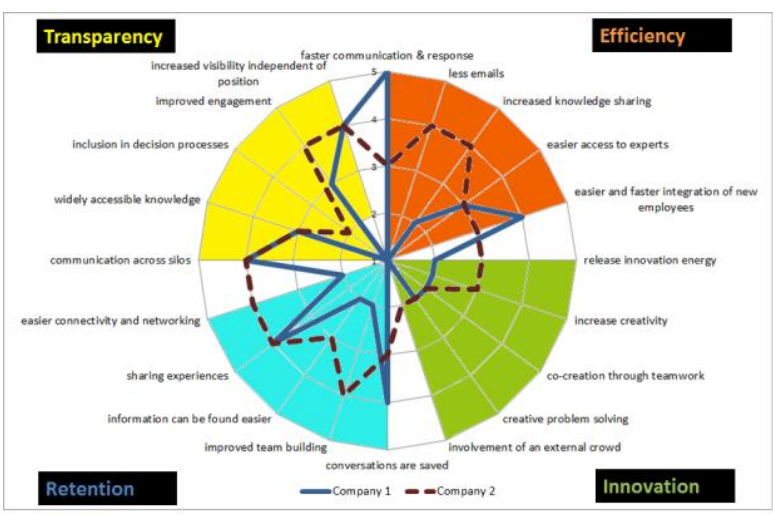

Figure 4: Comparing two organizations with the SMBV Compass 
Although company 1 obtained more points in the sub-category "faster communication \& response", company 2 performs better on all four dimensions. Company 1 has achieved 52 points if all dimensions are considered together, while company 2 has obtained 62 points. Both companies perform badly on the innovation dimension.

\section{Measuring the Business Value}

The business value of social platforms does not come from the platform itself but from how the platform is used within the company [3]. The business value of social platforms should be regularly measured in order to be aware not only of the current value, but also how the value changes over time. According to McKinsey survey, only 32 percent of executives report the use of quantitative measures to assess the impact of their social-technology use in their companies.[1]

In current practice, as underlined below, companies are not yet measuring the benefits derived from "social": "Actively measuring KPIs here, we do not do. But what you get with Slack is usage statistics, but you do not have an insight what is a good or a bad value. How many posts we had, who were the most active people, who posted how many messages, or how much happens in public channels and how much in direct messages, but it is not that we use these statistics actively."

As stated above, such simple business value measures can be captured using already existing features of the social platforms to follow key process indicators of activities taking place on the platforms. Analytics provide insights on how many users are active member of the platform, how many active channels are used and how many messages were sent in private or in public channels. However, as one manager pointed out: "I think the most important thing was to get colleagues (and customers) away from thinking that you can measure success by looking at basic activity such as \#users, \#documents posted, \#responses posted etc. I wanted to get them to start not from user activity, but from the question "what is my use case/what is my business objective?" and then think about measuring success in meeting that objective. If only 5\% of users are active, but your business saved money, your adoption is a success."

Different business value categories can be measured with different approaches, e.g., mail server logfile, content or social network analysis [17]. Furthermore, by assessing the business value, it can be seen if digital strategies to improve aspects of the business value have been successful. Examples of performance indicators are illustrated in the literature [6], which are elaborated here based on the interviews.

\subsection{Measuring Efficiency}

The first dimension, efficiency, can be measured for example by comparing the number of emails sent before and after the company-wide introduction of social platforms. This can be done with logfile analysis on the mail server but also via an evaluation of the number of posts and type of content. A successful usage of the platform will lead to a significant reduction of emails used internally and an increased satisfaction of employees: "[Email] is mostly externally. So it means all the internal updates, as we in the management team are sending information to people, it is most of the time done through Slack anyway."

Additionally, average response time on posts can help to measure efficiency of communication. The number of searches associated with a topic may indicate how often the digitized content accessed proves to be useful, or how interesting it is for the others. Associated ratings could be attached to evaluate the quality of content.

\subsection{Measuring Innovation}

Innovation can be measured in terms of the number and content of ideas that are created on the platforms. Qualitative content analysis could be applied to identify these ideas. Additionally, interviews or surveys could be conducted to ask employees about creativity and innovative idea generation using the wisdom of the crowd as described earlier. The wisdom of the crowd is enhanced through network effect, and the following four elements mentioned by Surowiecki: diversity, independence, decentralization and aggregation [18]. Thus, the size of the social network or the number of participants in certain channels may also be a good indicator of future innovation. As described above, in the Innovation Jam, different forms of text analytics can be associated to select the "prominent" ideas or select the valuable ideas. Additionally, other participants can "like" good contributions. Thus, the most liked ideas can be further developed.

\subsection{Measuring Retention}

As stated earlier, retention has two dimensions: retention of knowledge and retention of employees. A higher retention of employees can be achieved by a higher connectivity between employees. The value can be measured by the number of network connections (social network analysis) or the average number of followers. If these numbers increase over time, a higher connectivity between employees is achieved. The mood in the company or teams can be measured by 
positive and negative opinions expressed in the posts. Using sentiment analysis, the mood of employees or general sentiment can be automatically analyzed. Associated interventions may mitigate the diffusion of bad mood or identify issues to be addressed in order to improve the quality of the working environment. In order to measure the retention of knowledge, the number of posts and searches can be counted.

\subsection{Measuring Transparency}

For transparency, the number of open channels compared to the number of private channels shows how communication flows through the company. Furthermore, it can be measured how many posts are sent to employees from other departments, and how often people from different hierarchy levels interact. The number of likes and comments on a post, as well as the number of readers, give an indication how actively the social platforms are used, measure engagement of employees and reputation.

\section{Recommendations for Managers}

This article discusses the business value of social platforms and proposes the SMBV compass as a tool that enables managers to assess and better manage their digital knowledge work. Furthermore, it allows managers to orient their digital strategy in line with specific goals to better manage knowledge work using enterprise social platforms. In order to align organizational goals with their digital strategy we make the following three recommendations:

\section{Support and train your digital workforce:}

Management should actively support the usage of social platforms. Leaders can propel the uptake of social platforms by doing "the walk and talk", if they are convinced of the strategic business value or the potential value for gaining higher efficiency and innovation capabilities in the organization. Communicate the benefits of using social platforms and publish success stories or provide good examples, so that employees can directly understand the possibilities and the value of such platforms.

Train your workforce to harness technology and thus ensure that employees are aware of how to access the key functionality of the enterprise social platforms. It should be clear which tools should be used for which purpose. Showcase to new employees how to integrate the platforms in daily working routines. Ensure that employees know when to use such platforms and/or when to use another tool. These platforms should be used in a unified way in order to avoid confusion and messiness in the workplaces. Employees should not only be comfortable in skills that support the knowledge work but also understand the associated risks and interventions.

\section{Create an organizational culture that empowers employees to share and engage:}

An open culture that fosters informal interaction and communication is required in order to promote collaboration, knowledge sharing and successful use of the platforms. Organizational culture and norms should favor horizontal communication and reward knowledge-sharing behaviors and the contributors to the organizational knowledge. Steering of employees at group level (project team, department) may take place on a day-to-day basis through direct interaction with group members or managers. Social media management at the organizational level requires guidelines and policy setting. However, employees can self-regulate participation and be involved to contribute to the establishment of guidelines. Furthermore, both extrinsic and intrinsic motivations play an important role in knowledge sharing using social media in organizational contexts.

\section{Assess and boost the benefits of enterprise social platforms:}

Assess the business value and steer the usage of the platforms in your company according to specific goals and an associated strategy. Data from social platforms can be used in multiple ways to enhance business value for the company. As described earlier, by collecting usage statistics of the platforms, sentiment analysis or content analysis of textual data, business value can be measured. Algorithms and business intelligence services would further make it possible to derive summaries from discussions or ideas generated on the platform. The information overload can be reduced and additional business value can be generated. The SMBV compass (as presented in Figure 1) guides managers to align their social media strategy with strategic goals. Benefits can be reached if the results of the data analysis are integrated in the strategic decisions of the organization.

\section{Conclusions}

This article discusses the business value of enterprise social platforms and provides an overview of their main dimensions through the social media business value compass. The internal usage of social platforms may have four strategic purposes: (1) to improve efficiency, (2) to foster innovation, (3) to improve retention or (4) to increase the transparency of communication.

Our study found that few companies are currently assessing the business value of social media with con- 
crete measures (e.g. KPIs), and if they do, they do so only at very basic levels using simple analytics and statistics. The SMBV compass can provide a basis for managers in further developing their social media strategy and digital transformation.

Enterprise social platforms have the potential to drive efficiency, lead to new work practices and restructure the whole organization, but they do not necessarily change work practices or organizational culture. Enterprise social platforms can be introduced for different purposes: to improve efficiency, to foster team building, networking and collaboration, or to foster creativity and innovation. However, the way the digital workforce uses such technologies is embedded in the type of organization, its culture, the associated workflows, routines, and the knowledge processes associated with their tasks.

\section{References}

[1] Bughin, J., M. Chui, and M. Harrysson, "Transforming the business through social tools", McKinsey Survey, 2015. https://www.mckinsey.com/industries/high-tech/our-insights/ transforming-the-business-through-social-tools

[2] Bughin, J., L. LaBerge, and A. Mellbye, "The case for digital reinvention", McKinsey Quarterly February, 2017. https://www.mckinsey.com/business-functions/digitalmckinsey/our-insights/the-case-for-digital-reinvention

[3] Culnan, M.J., P.J. McHugh, and J.L. Zubillaga, "How large U.S. companies can use Twitter and other social media to gain business value", MIS Quarterly Executive 9(4), 2010, pp. 243-259.

[4] Grant, A., "In the Company of Givers and Takers", Harvard Business Review 91(4), 2013, pp. 90-97.

[5] Harrison, M., D. Schoder, and A. Tavakoli, "The evolution of social technologies", McKinsey Quarterly, 2016. https://www.mckinsey.com/industries/high-tech/our-insights/ the-evolution-of-social-technologies

[6] Herzog, C., A. Richter, M. Steinhüser, U. Hoppe, and M. Koch, "Methods and metrics for measuring the success of Enterprise Social Software - what we can learn from practice and vice versa", Proceedings of the 21st European Conference on Information Systems (ECIS 2013), (2013), 112.

[7] Kane, G.C., "Enterprise Social Media: Current Capabilities and Future Possibilities", MIS Quarterly Executive 14(1), 2015, pp. 1-16.

[8] Kappelman, L., V. Johnson, C. Maurer, et al., "The 2017 SIM IT-issues and trends study", MIS Quarterly Executive
17(1), 2018, pp. 53-88.

[9] Lam, H.K.S., A.C.L. Yeung, and T.C.E. Cheng, "The impact of firms' social media initiatives on operational efficiency and innovativeness", Journal of Operations Management 47-48, 2016, pp. 28-43.

[10] Larson, K., and R. Watson, "The value of social media: toward measuring social media strategies", Thirty Second International Conference on Information Systems, (2011), 118.

[11] Leidner, D.E., E. Gonzales, and H. Koch, "An affordance perspective of enterprise social media and organizational socialization", Journal of Strategic Information Systems 27(2), 2018, pp. 117-138.

[12] McAfee, A., Enterprise 2.0: New collaborative tools for your organization's toughest challenges, Harvard Business Press, 2009.

[13] Razmerita, L., K. Kirchner, and T. Nabeth, "Social Media in Orgamizations: Leveraging Personal and Collective Knowledge Processes", Journal of Organizational Computing and Electronic Commerce 24(1), 2014, pp. 7483.

[14] Razmerita, L., K. Kirchner, and P. Nielsen, "The Perceived Business Value of Social Media at Work", 5th MSphere Conference 2016, (2016), 1-15.

[15] Razmerita, L., K. Kirchner, and P. Nielsen, "What factors influence knowledge sharing in organizations? A social dilemma perspective of social media communication", Journal of Knowledge Management 20(6), 2016, pp. 12251246.

[16] Razmerita, L., and C.-W. Tan, "Disentangling the Effects of Collaborative Social Platforms on Organizational Knowledge Practices and Innovation: A Mixed-Methods Study of a Non-Profit Organization", 5th pre-ICIS International Workshop on the Changing Nature of Work (CNoW), (2017).

[17] Richter, A., J. Heidemann, M. Klier, and S. Behrendt, "Success Measurement of Enterprise Social Networks", International Conference on Wirtschaftsinformatik, (2013), $1-15$.

[18] Surowiecki, J., The wisdom of crowds: Why the many are smarter than the few and how collective wisdom shapes business, Random House, Doubleday Books, New York, 2004.

[19] Wehner, B., T. Falk, and S. Leist, "What Benefits Do They Bring? a Case Study Analysis on Enterprise Social Networks", ECIS 2017, (2017), 2069-2085. 